\title{
HIGH POWER TEST OF THE ISAC TRIPLE GAP BUNCHER OPERATING IN CW MODE
}

\author{
Y. V. Bylinsky, A. I. Kvasha, A. A. Menshov, P. N. Ostroumov, V. V. Paramonov, INR RAS \\ G. Dutto, R. E. Laxdal, A. K. Mitra, R. L. Poirer, TRIUMF
}

\begin{abstract}
A separated function, variable energy, drift tube linac (DTL) operating in cw mode at $105 \mathrm{MHz}$ is being built for the ISAC radioactive beam facility at TRIUMF. Included in the design are three buncher cavities installed in the first, second and third drift spaces between the five IH tanks. The main requirements of the bunchers are: $\mathrm{cw}$ mode, high effective voltage (up to $0.32 \mathrm{MV}$ ), large velocity acceptance (1.8-4.1\%). The first DTL buncher has been developed at INR and is being tested at TRIUMF. It is a triple gap split-ring rf structure operating at $105 \mathrm{MHz}$. Signal level measurements give a Q-value of 4300 which is $74 \%$ of MAFIA simulation predictions and a resonant frequency within $0.6 \%$ of the calculated value. With cooling water flow of $20 \mathrm{l} / \mathrm{min}$ the mechanical vibrations were measured to be of the order of $1 \mu \mathrm{m}$. Although the design parameters are $56 \mathrm{kV}$ gap voltage and $8 \mathrm{~kW}$ nominal power, we demonstrated $85 \mathrm{kV}$ and $16 \mathrm{~kW}$ respectively with stable operation.
\end{abstract}

\section{INTRODUCTION}

Buncher is designed as a triple gap split-ring rf structure operating at $105 \mathrm{MHz}$ in $\mathrm{cw}$ mode. Buncher specifications are given in Table 1 and described in details elsewhere [1]. The effective voltage $V_{\text {eff }}$ is quoted for the particle design velocity.

Table 1: Summary of parameter specifications for triple gap Split-ring buncher

\begin{tabular}{|l|l|l|}
\hline Frequency & $\mathrm{f}$ & $105 \mathrm{MHz}$ \\
\hline Particle design velocity & $\beta_{0}$ & 0.023 \\
\hline Effective voltage & $\mathrm{V}_{\text {eff }}$ & $190 \mathrm{kV}$ \\
\hline Drift tube voltage & $\mathrm{V}_{\text {}}$ & $56 \mathrm{kV}$ \\
\hline
\end{tabular}

Detailed study based on the MAFIA 3-D simulations has been performed in order to meet buncher specifications [2]. A series of various measurements has been conducted at TRIUMF to ensure a stable and reliable device operation. Basing on these results two other DTL bunchers will be designed and manufactured in a year from now.

\section{MECHANICAL DESCRIPTION}

A general view of the buncher structure is shown in Figure 1. Buncher basic dimensions are presented in Table 2.

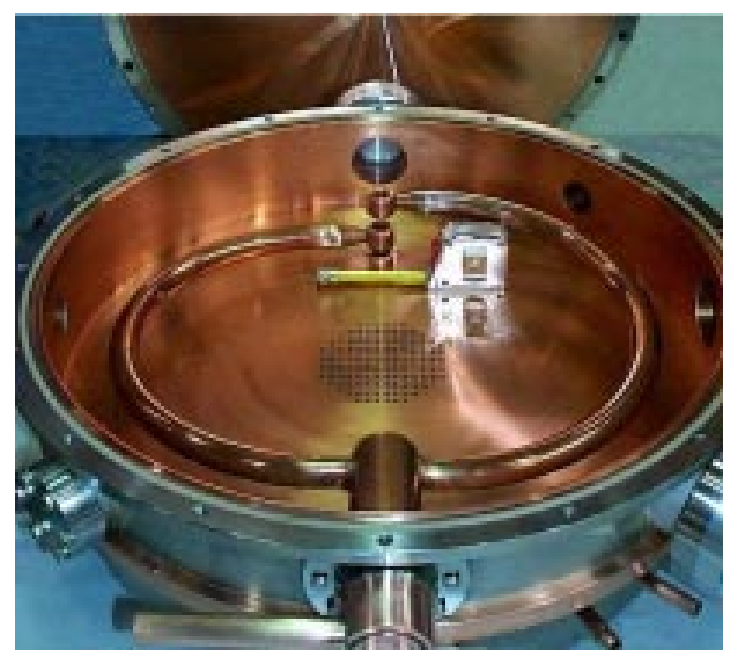

Figure 1: General view of the Split-ring buncher.

Table 2: Split-ring buncher basic dimensions

\begin{tabular}{|l|l|l|}
\hline Tank diameter & {$[\mathrm{mm}]$} & 550 \\
\hline Tank length & {$[\mathrm{mm}]$} & 98 \\
\hline Ring bending curvature radius & {$[\mathrm{mm}]$} & 175 \\
\hline Ring tube diameter & {$[\mathrm{mm}]$} & 25 \\
\hline Aperture diameter & {$[\mathrm{mm}]$} & 14 \\
\hline Drift tube diameter & {$[\mathrm{mm}]$} & 30 \\
\hline
\end{tabular}

Split-ring is made of copper tube. 2 half rings, which carry the drift tubes, are mounted on a supporting leg. Rings are declined in beam direction in order to bring ring tips right at the corresponding drift tube centres. Half-rings contain straight cylindrical extensions at the bottom and 2 short cones on top to support the drift tubes. Straight cylindrical part and cones serve for the coarse frequency tuning. The rings are water cooled.

A capacitive frequency tuner is dedicated for fine tune of the buncher fundamental frequency and to operate as an executive body in the frequency feedback loop of the rf control system. It is a cooper plate with $55 \mathrm{~mm}$ in diameter, supported by the rod. An automatic mechanical actuator allows $40 \mathrm{~mm}$ movement, brining the plate to the drift tubes as close as $10 \mathrm{~mm}$. Tuner is not cooled.

Buncher is fed through a coupling loop, attached to a standard transmission line (3.125”). A ceramic tube serves as an rf vacuum window. Window area is cooled, while the loop itself is not.

Both cooper buncher body and stainless steel lid are water cooled. 


\section{RF SIGNAL LEVEL TEST}

First frequency measurement gave $600 \mathrm{kHz}$ offset from the MAFIA prediction. The coarse frequency tuning was done by means of ring length correction. A bead-pull measurement allowed reconstruction of the electric field distribution along the beam axis as well as shunt impedance evaluation. Figure 2 shows experimental points in comparison with SUPERFISH simulated data for the accelerating field distribution.

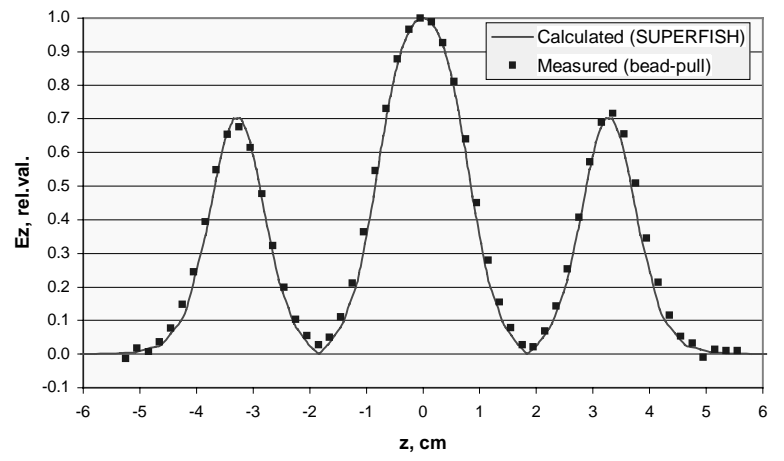

Figure 2: Accelerating field distribution.

The most general rf parameters are presented in Table 3 in comparison with figures obtained from MAFIA simulations.

Table 3: Buncher general rf parameters.

\begin{tabular}{|l|l|l|}
\hline Parameter & Calc. & Meas. \\
\hline Design velocity, $\beta_{0}$ & $2.3 \%$ & - \\
\hline Operating frequency, $\mathrm{MHz}$ & 104.6 & 105.2 \\
\hline Frequency tuning range, $\mathrm{kHz}$ & 900 & 1200 \\
\hline Nearest mode frequency, $\mathrm{MHz}$ & 102.7 & 103.5 \\
\hline $\mathrm{Q}-$ factor & 5800 & 4300 \\
\hline Shunt impedance $\mathrm{R}_{\mathrm{s}}^{*}, \mathrm{M} \Omega$ & 9.16 & 6.15 \\
\hline $\mathrm{R}_{\mathrm{s}}^{*} / \mathrm{Q}, \Omega$ & 1580 & 1430 \\
\hline Maximum surface field, $\mathrm{MV} / \mathrm{m}$ & 13.2 & - \\
\hline Nominal power, $\mathrm{kW}$ & 5.5 & $\sim 8.1$ \\
\hline
\end{tabular}

$\mathrm{R}_{\mathrm{s}}^{*}=\mathrm{V}^{2} / \mathrm{P}, \mathrm{V}=4 \mathrm{~V}_{1}, \mathrm{~V}_{1}$ - peak voltage across first accelerating gap, $\mathrm{P}-$ dissipated rf power.

A delicate study has been performed in order to determine buncher mechanical stability. Basically, the Split-ring structure can be considered as two identical curved cantilever beams, fixed at one end and free to vibrate at the other. Ring mechanical oscillations have been measured with a dynamic signal analyzer (HP \#35665A) and two accelerometers attached to the drift tube. Table 4 presents measurement results for the most sensitive direction (lateral axis). Here induced oscillations were caused by the water flow of $20 \mathrm{l} / \mathrm{min}$. Measurements show that vibration amplitude is much below the drift tube displacement tolerance of $0.2 \mathrm{~mm}$.
Table 4: Split-ring mechanical vibration parameters in the lateral direction.

\begin{tabular}{|l|l|l|l|l|l|l|}
\hline Frequency & $\mathrm{MHz}$ & 11 & 36 & 39 & 96 & 107 \\
\hline Amplitude & $\mu \mathrm{m}$ & 1 & 1 & 1 & 0.1 & 0.1 \\
\hline
\end{tabular}

\section{HIGH POWER TEST RESULTS}

A $105 \mathrm{MHz}$ amplifier with output power up to $20 \mathrm{~kW}$ was used as a buncher rf power supply. A flexible stable phase coaxial line with air insulation served as an rf transmitting line. A high power phase shifter (trombone) was implemented to $\mathrm{rf}$ feeding line in order to remove waveguide parasitic modes from the fundamental frequency.

High power rf test was preceded with delicate vacuum test and conditioning. Surface outgassing was forced with bake out at $50^{\circ}-60^{\circ} \mathrm{C}$. Following 10 hours of vacuum conditioning a base pressure of $2 \cdot 10^{-7}$ Torr was achieved.

A low level $(<100 \mathrm{~W})$ rf conditioning was one of the longest stages ( $\sim 7$ hours) on the way to the buncher nominal power. It was accompanied with electron multipactor discharge and intense outgassing. Following voltage rise was restricted by high voltage discharges which were initiated when vacuum dropped to $\sim 2 \cdot 10^{-6}$ Torr. Nominal voltage was obtained in 6 hours with intermediate half-power conditioning for 26 hours during weekend. Rf conditioning was performed in $\mathrm{cw}$ mode because pulsed mode eventually appears to be ineffective. Long-term conditioning has brought a significant vacuum improvement: from $\sim 1 \cdot 10^{-6}$ Torr to $\sim 3 \cdot 10^{-7}$ Torr at the full power. And although the design parameters are $56 \mathrm{kV}$ gap voltage and $\sim 8 \mathrm{~kW}$ nominal power, we eventually achieved $85 \mathrm{kV}$ and $16 \mathrm{~kW}$ respectively with stable operation. After conditioning it took couple of hours to put buncher under full power after short term vacuum brake and $\sim 15$ minutes from standby mode. The longest continuous run at nominal voltage lasted for 80 hours. Taking into account a number of various runs from 5 to 20 hours duration, the buncher operated stable for $\sim 250$ hours in total.

Rf conditioning has left multipactor discharge traces on the cavity and endplate walls in a small area around the leg. But finally buncher operated without multipactoring in the voltage range from $10 \%$ to the highest level.

Figure 3 demonstrates typical dependencies of rf power and residual gas pressure upon the drift tube voltage. Vacuum drop at low rf voltage level vanished after the buncher rf conditioning.

In order to double check the voltage level, achieved in the buncher, a calibration of the drift tube voltage has been performed. It was based on the measurement of the energy of the $\mathrm{x}$-rays, produced with free electrons accelerated in the buncher electric field. Measurement showed very good agreement with pickup loop data, based on the shunt impedance figure. 


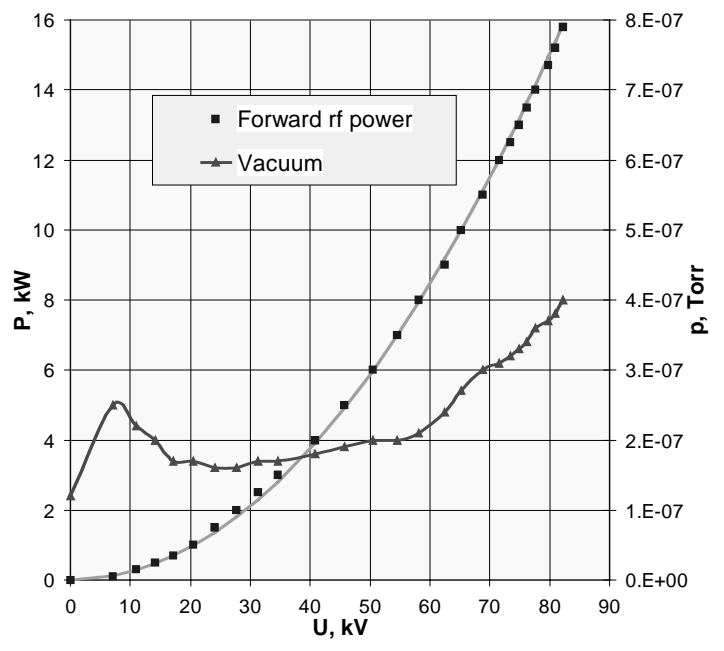

Figure 3: Rf power and vacuum vs drift tube voltage.

Measurements of the operating frequency dependence upon rf power showed frequency increasing with Splitring heating (see Figure, 4 curve 2), while opposite tendency (see Figure 4, curve 1) has been observed with rf off and structure heating by the water passing through the buncher cooling channels.

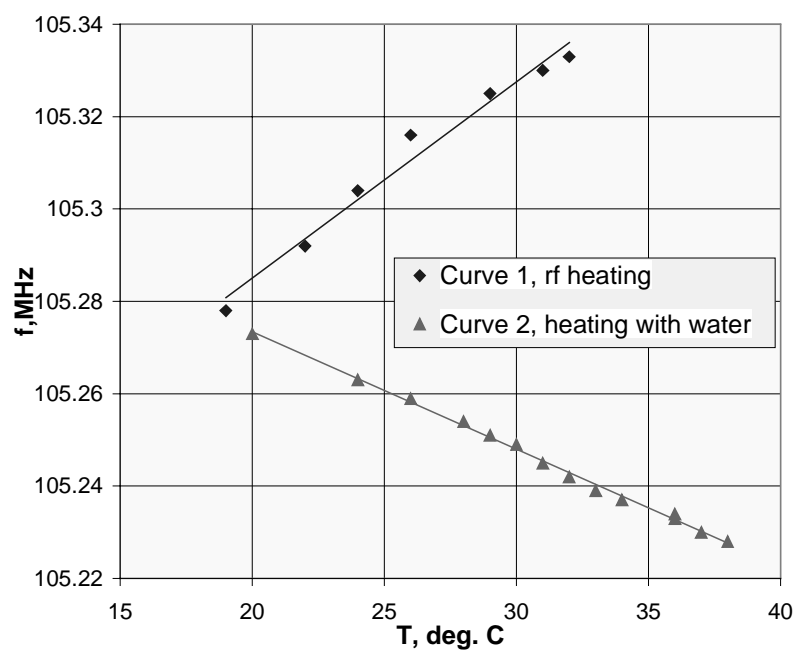

Figure 4: Buncher frequency vs Split-ring temperature.

This unexpected frequency behaviour can be explained with non-uniform thermal load due to rf losses in the Split-ring structure. It can cause a deformation of the ring. MAFIA simulations confirm this phenomena of the frequency increasing assuming small movement of the rings along beam direction. In fact ring deformation take place in the buncher. Thus drift tube transverse movement due to rf thermal load of the ring was measured using a telescope on targets installed in the drift tubes. Initially drift tube displacement from the beam axis reached 580 $\mu \mathrm{m}$ for nominal rf power. Then it was reduced to $230 \mu \mathrm{m}$ with increasing of the cooling water flow in the Split-ring from 13 to $35 \mathrm{l} / \mathrm{min}$. Another reduction of the drift tubes movement by factor 2 was achieved by reversing the water flow direction to cool the hottest ring area first.

High surface electric fields, up to $13 \mathrm{MV} / \mathrm{m}$, initiate electron emission. Those electrons accelerated in the electric field knock to the cavity walls and other elements of structure producing x-rays. Radiation field measurement at the buncher surface showed level over $1000 \mathrm{mS} / \mathrm{h}$ for the full rf power. Thus additional lead shielding has been implemented to allow acceptable working conditions at the test stand.

Charged particle current produced and accelerated in the buncher was detected in the diagnostics box, which is attached to the buncher. Field emission is considered to be the nature of this current. Observed current is bunched at the fundamental frequency with an intensity of tens of $\mu \mathrm{A}$ for the nominal buncher setting. This current is intense enough to overwhelm signals from the desired beam of ions and even harm the delicate beam diagnostic devices. Special measures should be foreseen to avoid this problem.

During the high power tests both: tuner and coupling loop demonstrated very reliable performance in the whole working range without any indication of sparks or multipactoring.

\section{CONCLUSION}

The $105 \mathrm{MHz}$ Split-ring buncher showed stable and reliable rf performance under full power $(8 \mathrm{~kW})$ and much higher (up to $16 \mathrm{~kW}$ ). No multipactor discharge has been observed in the specified voltage region.

Split-ring structure design appears to be rather stable against mechanical vibrations. Measured vibration amplitude of $\sim 1 \mu \mathrm{m}$ is two orders lower than tolerated value. However considerable movement of the drift tubes $(\sim 500 \mu \mathrm{m})$ due to rf thermal load of the ring has been observed. It could be reduced below tolerance of $200 \mu \mathrm{m}$ with more effective cooling.

Essential x-ray field was measured around the buncher especially over the nominal voltage. It is caused by the electrons, produced in the buncher volume due to field emission and accelerated in the electrical field. Bunched electron current of tens of $\mu \mathrm{A}$ was measured on the beam axis at the buncher entrance. This could be a grate concern for the DTL bunchers \#2 end \#3 as their voltage specification is much higher then for the first one.

\section{REFERENCES}

[1] R. Laxdal, The Separated Function Drift Tube Linac for ISAC, TRI-DN-97-4, TRIUMF Design Note, April 1997.

[2] Y. Bylinsky et al., A Triple Gap Resonator Design for the Separated Function DTL at TRIUMF, Proc. Of the PAC-97, Vancouver, May 1997, pp. 1135-1137. 